\title{
Quantal Brownian Motion - Dephasing and Dissipation
}

\author{
Doron Cohen \\ Department of Physics of Complex Systems, \\ The Weizmann Institute of Science, Rehovot 76100, Israel. \\ (Published in Journal of Physics A 31, 8199-8220 (1998)))
}

\begin{abstract}
We analyze quantal Brownian motion in $d$ dimensions using the unified model for diffusion localization and dissipation, and Feynman-Vernon formalism. At high temperatures the propagator possess a Markovian property and we can write down an equivalent Master equation. Unlike the case of the Zwanzig-Caldeira-Leggett model, genuine quantum mechanical effects manifest themselves due to the disordered nature of the environment. Using Wigner picture of the dynamics we distinguish between two different mechanisms for destruction of coherence: scattering perturbative mechanism and smearing non-perturbative mechanism. The analysis of dephasing is extended to the low temperature regime by using a semiclassical strategy. Various results are derived for ballistic, chaotic, diffusive, both ergodic and non-ergodic motion. We also analyze loss of coherence at the limit of zero temperature and clarify the limitations of the semiclassical approach. The condition for having coherent effect due to scattering by lowfrequency fluctuations is also pointed out. It is interesting that the dephasing rate can be either larger or smaller than the dissipation rate, depending on the physical circumstances.
\end{abstract}

\section{Introduction}

Classical Brownian motion is described by the Langevin equation

$$
m \ddot{\mathbf{x}}+\eta \dot{\mathbf{x}}=\mathcal{F}
$$

where $\mathbf{x}$ is the position of the particle, $\eta$ is the friction coefficient, and $\mathcal{F}=-\nabla \mathcal{U}(\mathbf{x}, t)$ is a stochastic force. This equation is meaningful only in a statistical sense. The time evolution of a phase space distribution $\rho(\mathbf{x}, \mathbf{p})$ is obtained by solving (1) for various realizations of the stochastic potential, and then averaging over all these realizations. The stochastic potential is zero on the average and its correlations are

$$
\left\langle\mathcal{U}\left(\mathbf{x}^{\prime \prime}, t^{\prime \prime}\right) \mathcal{U}\left(\mathbf{x}^{\prime}, t^{\prime}\right)\right\rangle=\phi\left(t^{\prime \prime}-t^{\prime}\right) \cdot w\left(\mathbf{x}^{\prime \prime}-\mathbf{x}^{\prime}\right)
$$

Typically these correlations are characterized by a 'short' temporal scale $\tau_{c}$ and a 'microscopic' spatial scale $\ell$. Usually, it is further assumed that higher moments are determined by Gaussian statistics. The Langevin description can be derived by considering a general Hamiltonian of the form

$$
\mathcal{H}=\mathcal{H}_{0}(\mathbf{x}, \mathbf{p})+\mathcal{H}_{\text {env }}\left(\mathbf{x}, Q_{\alpha}, P_{\alpha}\right)
$$

where $\mathbf{x}$ and $\mathbf{p}$ are the canonical variables that correspond to the distinguished degree of freedom, $\mathcal{H}_{0}=\mathbf{p}^{2} / 2 m$ is the free motion Hamiltonian, and $\left(Q_{\alpha}, P_{\alpha}\right)$ are 
environmental degrees of freedom. The dynamical variable $\mathbf{x}$ may represent the position of a large particle. In the one-dimensional version of Brownian motion it may represent the position of a piston. The actual conditions for having the reduced Langevin description turn out to be quite weak [1]. The environment should consist of at least 3 degrees of freedom with fast chaotic dynamics. Fast implies that the classical motion is characterized by a continuous spectrum with high frequency cutoff, such that the motion of the environment can be treated adiabatically with respect to the slow motion of the particle. It is essential to assume that the following condition is fulfilled

$$
\text { Generic Brownian Motion } \Leftrightarrow \quad \frac{v}{\ell} \ll \frac{1}{\tau_{c}}
$$

where $\tau_{c}$ and $\ell$ characterize the correlations of the stochastic potential which is experienced by the particle. Eq.(4) is the condition for using the white noise approximation (WNA). namely, the noise is characterized by its intensity

$$
\nu \equiv \int_{-\infty}^{\infty} \phi(\tau) d \tau \cdot\left|w^{\prime \prime}(0)\right|
$$

and its temporal correlations are adequately described by the formal expression $\phi(\tau)=\nu \delta(\tau)$. Without loss of generality we assume from now on the normalization $w^{\prime \prime}(0)=-1$. The correlations of the stochastic force satisfy

$$
\left\langle\mathcal{F}(t) \mathcal{F}\left(t^{\prime}\right)\right\rangle_{\text {at }} x=\phi\left(t-t^{\prime}\right)
$$

In the general case, (6) is less informative than (2). However, in case of a classical particle that experiences white noise, the additional information is not required at all! It should be emphasized that this observation does not hold if (偖) is not satisfied: If $\tau_{c}$ is larger than $\ell / v$, then the particle will perform a stochastic motion that depends crucially on the "topography" of the stochastic potential.

The classical analysis further reveals [1] that associated with the stochastic potential there is also a dissipation effect. If the environment is characterized by either micro-canonical or canonical temperature $T$, then the friction parameter will be

$$
\eta=\frac{\nu}{2 k_{B} T} \quad \text { Fluctuations } \leadsto \text { Dissipation }
$$

Thus, any generic environment, in the sense specified above, leads to the universal ohmic behavior. The motion of the particle is determined by the interplay between the friction and the noise. The friction leads to damping of the particle's velocity, while the noise pumps energy back into its motion. Eventually we have diffusion with coefficient $D_{\eta}=\nu / \eta^{2}$. If (4) is not satisfied, then we will have diffusion even in the absence of dissipation. The latter "non-dissipative diffusion" is characterized by the coefficient $D_{0} \sim \ell v$, where $v$ is the velocity of the particle. This latter type of diffusive behavior should not be confused with the generic "dissipative diffusion" that characterizes Brownian motion.

One wonders whether there is a well defined quantized version of the above Langevin equation. Is it possible, quantum-mechanically, to characterize a universal dynamical behavior that corresponds to classical Brownian motion? Are stronger physical conditions required in order to guarantee generic behavior? In the present paper we have no intention to give a full answer to all these questions. Rather, as in previous publications [2], we follow the Caldeira-Leggett strategy [3]. Namely, we consider the motion of a particle under the influence of an effective (non-chaotic) bath 
that is composed of infinitely many oscillators. The proper model that corresponds (classically) to the Langevin equation (11) with (2) is the DLD model that has been introduced in [2]. DLD are the initials of "Diffusion Localization and Dissipation". These three effects comes out naturally from the quantum-mechanical solution of the DLD model.

Quantum mechanically, it is convenient to use Wigner function $\rho(R, P)$ in order to represent the reduced probability density matrix. We can use the Feynman-Vernon $(\mathrm{FV})$ formalism [4] in order to find an expression for the propagator $\mathcal{K}\left(R, P \mid R_{0}, P_{0}\right)$. This propagator is uniquely determined once the friction $(\eta)$, the temperature of the bath $(T)$ and the spatial correlations $w(r)$ are specified. The dependence of the propagator on the bath-temperature is via the appearance of a quantummechanical version of the noise kernel $\phi(\tau)$. The latter is related to the friction coefficient via a universal fluctuation-dissipation relation. At high temperatures the quantum-mechanical $\phi(\tau)$ coincides with its classical expression. At the limit of zero temperature $\phi(\tau)$ does not vanish, rather it develops a large negative tail. In the latter case, $\phi(\omega)$, the power spectrum of the noise, reflects the zero point fluctuations (ZPF) of the environmental modes.

The Zwanzig-Caldeira-Leggett (ZCL) model [3] constitutes a special formal limit of the DLD model. It is obtained by taking the limit $\ell \rightarrow \infty$. Both the ZCL model and the DLD model will be generalized in this paper to allow the analysis of Brownian motion in $d$-dimensions. A modified version of the the DLD model incorporates the effect of long range (power-law) spatial correlations. On the other hand, we are not discussing the DLD model in its full generality (as in [2]), but rather restricting ourselves to the particular circumstances that correspond in the classical limit to generic Brownian motion. Thus, we are considering the ohmic DLD model, and further assume that (4) is satisfied.

In case of the ZCL model, the quantal propagator $\mathcal{K}\left(R, P \mid R_{0}, P_{0}\right)$ is a Gaussian stochastic kernel. Consequently the dynamics can be obtained by solving the Langevin equation (11) with (6). At low-temperatures the quantum mechanical version of $\phi(\tau)$ should be used. At high temperatures $\phi(\tau)$ becomes classical-like and consequently the ZCL propagator coincides with its classical limit. These observations do not hold in case of the DLD model [2]. Furthermore, the distinction between the quantal DLD propagator and its classical limit persists even in the limit of high temperatures.

It is important to define what is the meaning of 'high temperatures'. As in the classical case, a relatively simple description of the dynamics is obtained if it possesses a Markovian property. With a Markovian property it is possible to obtain the longtime evolution by composing short-time evolution steps. It is also possible then to write down a corresponding Master equation for the Wigner function. The FV pathintegral expression for the propagator contains the quantum-mechanical version of $\phi(\tau)$ rather than the classical one. In order to have a Markovian property one should argue that it is possible to use the WNA, meaning to replace $\phi(\tau)$ by the effective classical-like delta-function. Still, in case of the DLD model, the result of the path integration is not classical-like: The high-temperature Markovian limit is not the same as the classical limit.

It turns out that the quantum-mechanical condition for using the WNA, thus having a Markovian property, is more restrictive than (4). For ballistic-like motion we shall see that the actual condition is, in most circumstances,

$$
\text { High Temperatures } \Leftrightarrow \frac{v}{\ell} \ll \frac{k_{B} T}{\hbar}
$$


A recent derivation of the high temperature ohmic DLD model which is based on a synthetic RMT Hamiltonian has been reported in [5]. The existence of such a derivation is most encouraging since it further support the idea of having a universal description of quantal Brownian motion, at least in the Markovian limit. It has been speculated by the authors of the latter reference that a future extension of their formalism will lead to an agreement with the general result of [2]. The high temperature ohmic DLD model has been discussed in [2] and has been further analyzed in [5]. The physical picture of high-temperature dynamics will be further illuminated in the present paper.

Loss of coherence, or dephasing, is a central issue in the analysis of quantal Brownian motion. Wigner's picture of the dynamics leads to the distinction between two different mechanisms for loss of coherence. In case of ZCL model one should consider the 'spreading mechanism'. This classical-like non-perturbative mechanism is very effective in smearing away the interference pattern. In case of the DLD model, coherence is much better maintained, and one should consider the perturbative 'scattering mechanism' for dephasing.

At the limit of zero temperature, the spreading mechanism is still effective in suppressing interference. One can use the Langevin formalism in order to analyze the smearing of the interference pattern. It is important, however, to take into account the negative temporal-correlations of the effective noise [6]. On the other hand, the analysis of low-temperature dephasing in case of the 'scattering mechanism' is a quite subtle issue, that constitutes a main concern of the present paper. The Langevin formalism is no-longer applicable, and the lack of a Markovian property enforce a semiclassical approach. The semiclassical approach has a further advantage: it is possible to go beyond the analysis of a simple ballistic-like Brownian motion and to analyze other types of transport. We shall distinguish between ballistic, diffusive and chaotic motions through cavities. We shall derive various results and contributions to the dephasing rate in the various temperature regimes and depending on the physical circumstances. Some of these results coincide with similar computations that are related to electrons in metal [7].

An important question is whether the scattering mechanism is still effective in suppressing interference at the limit of zero temperature [8, 9]. It turns out that our semiclassical approach, in spite of its other advantages, has a limited range of validity. It can be trusted if the kinetic energy of the particle is sufficiently large. For ballistic-like motion large energy means

$$
\text { Large Energy } \Leftrightarrow \quad \frac{v}{\ell} \ll \frac{E}{\hbar}
$$

in analogy with (8). The latter condition can be cast into the more suggestive form $\lambda_{B} \ll \ell$, where $\lambda_{B}$ is the De-Broglie wavelength of the particle. The large-energy condition is obviously satisfied in case of the ZCL model $(\ell \rightarrow \infty)$. The large energy condition may not be satisfied in case of the DLD model, and consequently the semiclassical result should be modified. In particular, in case of a low-temperature thermal motion, the contribution of the zero point fluctuations (ZPF) to the dephasing rate should be excluded. 


\section{The ohmic DLD Model}

We consider a bath that consists of infinitely many oscillators whose canonical coordinates are $\left(Q_{\alpha}, P_{\alpha}\right)$. The bath Hamiltonian is

$$
\mathcal{H}_{\text {bath }}=\sum_{\alpha}\left(\frac{P_{\alpha}^{2}}{2 m_{\alpha}}+\frac{1}{2} m \omega_{\alpha}^{2} Q_{\alpha}^{2}\right)
$$

In case of the DLD model the interaction of the particle with the oscillators is described by

$$
\mathcal{H}_{\text {int }}=-\sum_{\alpha} c_{\alpha} Q_{\alpha} u\left(\mathbf{x}-\mathbf{x}_{\alpha}\right)
$$

where $\mathbf{x}_{\alpha}$ is the location of the $\alpha$ oscillator, $u\left(\mathbf{x}-\mathbf{x}_{\alpha}\right)$ describes the interaction between the particle and the $\alpha$ oscillator, and $c_{\alpha}$ are coupling constants. It is assumed that the function $u(\mathbf{r})$ depends only on $|\mathbf{r}|$. The range of the interaction will be denoted by $\ell$. The oscillators are distributed uniformly all over space. Locally, the distribution of their frequencies is ohmic. Namely,

$$
\frac{\pi}{2} \sum_{\alpha} \frac{c_{\alpha}^{2}}{m_{\alpha} \omega_{\alpha}} \delta\left(\omega-\omega_{\alpha}\right) \delta\left(\mathbf{x}-\mathbf{x}_{\alpha}\right)=\eta \omega \quad \text { for } \omega<1 / \tau_{c} .
$$

It is useful to define the spatial auto-correlation function

$$
w(\mathbf{r})=\int_{-\infty}^{\infty} u\left(\mathbf{r}-\mathbf{x}^{\prime}\right) u\left(\mathbf{x}^{\prime}\right) d x^{\prime}
$$

Without loss of generality $u(\mathbf{r})$ is normalized such that $w^{\prime \prime}(0)=-1$. For example, we may consider a Gaussian $u(\mathbf{r})$ for which

$$
w(\mathbf{r})=\ell^{2} \exp \left(-\frac{1}{2}\left(\frac{\mathbf{r}}{\ell}\right)^{2}\right)
$$

The $d$-dimensional FT of $w(\mathbf{r})$ will be denoted by $\tilde{w}(\mathbf{k})$. The mode-density (after angular integration) is $g(k)=\left(C_{d} /(2 \pi)^{d}\right) k^{d-1} \tilde{w}(k)$. In general, we shall assume that

$$
g(k)=C \ell^{2+\sigma} k^{\sigma-1} \quad \text { for } k<1 / \ell
$$

where $\ell$ characterize the spatial scale of the correlations, and $C$ is a dimensionless constant. In case of the short range Gaussian-type correlations (14), the parameter $\sigma$ equals simply to the dimensionality $d$. For long range power-law interaction it may be less than $d$, possibly negative. In a moment we shall argue that in order to have a well defined model we must have $\left|w^{\prime \prime}(0)\right|<\infty$. Therefore only $-2<\sigma$ is meaningful. The regime $-2<\sigma \leq 0$ is well defined but it requires special treatment since $w(0)$ diverges.

The formal limit $\ell \rightarrow \infty$ corresponds to the ZCL model. The ZCL model describes interaction of a particle with environmental modes whose wavelength is much larger compared with the distances that are explored by the particle. Consequently, the interaction with the $\alpha$ field-mode is approximated by a linear function. The interaction term in the Hamiltonian that defines the ZCL model is of the following form (here generalized to $d$-dimensions):

$$
\mathcal{H}_{\text {int }}=\sqrt{d} \sum_{\alpha} c_{\alpha} Q_{\alpha} \hat{n}_{\alpha} \cdot \mathbf{x}
$$

The unit vectors $\hat{n}_{\alpha}$ are assumed to be distributed isotropically. The distribution of the oscillators with respect to their frequencies is assumed to be ohmic, as in (12) with the $\delta\left(\mathbf{x}-\mathbf{x}_{\alpha}\right)$ omitted. 
The classical analysis of the $d$-dimensional DLD model constitutes a trivial generalization of the one-dimensional DLD model that has been considered in [2]. Equation (3.10) in the latter reference should be replaced by the following expression for the friction:

$$
\mathcal{F}_{\text {friction }}(\mathbf{v})=\int_{-\infty}^{\infty} \alpha(\tau) \nabla w(\mathbf{v} \tau) d \tau
$$

where $\mathbf{v}$ is the velocity of the particle. For ohmic bath $\alpha(\tau)$ can be replaced by $-\eta \delta^{\prime}(\tau)$. One obtains then $\mathcal{F}_{\text {friction }}=-\eta \mathbf{v}$. Obviously, in order to obtain a finite result it is crucial to have $\left|w^{\prime \prime}(0)\right|<\infty$. Recall that by convention we use the normalization $w^{\prime \prime}(0)=-1$. Thus, the generalized version (15) of the DLD model is well-defined provided $-2<\sigma$. The reduced motion of the particle will be described by the Langevin equation (11), where the noise satisfies (2), with $\phi(\tau)=2 \eta k_{B} T \delta(\tau)$. For the realization of a classical trajectory, the global functional-form of $w(\mathbf{r})$ is of no importance. Only the force correlations (6) are important. The latter correlations are well defined as long as $\left|w^{\prime \prime}(0)\right|<\infty$, and by our convention they are equal $\phi(\tau)$. The parameter $\ell$ is insignificant in the classical analysis of the ohmic DLD model, and therefore the classical description of Brownian motion is the same as in the case of the ZCL model.

Quantum mechanically, the reduced dynamics of the system may be described by the propagator $\mathcal{K}\left(R, P \mid R_{0}, P_{0}\right)$ of the probability density matrix. For sake of comparison with the classical limit one uses Wigner function $\rho(R, P)$ in order to represent the latter. Using Feynman-Vernon (FV) formalism [4, an exact pathintegral expression for this propagator is obtained. The FV expression is a double sum $\iint \mathcal{D} x^{\prime} \mathcal{D} x^{\prime \prime}$ over the path variables $x^{\prime}(\tau)$ and $x^{\prime \prime}(\tau)$. It is convenient to use new path variables $R=\left(x^{\prime}+x^{\prime \prime}\right) / 2$ and $r=\left(x^{\prime \prime}-x^{\prime}\right)$, and to transform the $\iint \mathcal{D} R \mathcal{D} r$ integral into the form [2]

$$
\mathcal{K}\left(R, P \mid R_{0}, P_{0}\right)=\int_{R_{0}, P_{0}}^{R, P} \mathcal{D} R \mathcal{K}[R]
$$

where $\mathcal{K}[R]$ is a real functional, which is defined by the expression:

$$
\mathcal{K}[R]=\int \mathcal{D} r \quad \mathrm{e}^{i \frac{1}{\hbar}\left(S_{\text {free }}+S_{F}\right)} \mathrm{e}^{-\frac{1}{\hbar^{2}} S_{N}} .
$$

The $\mathcal{D} r$ integration is unrestricted at the endpoints, and the free action functional is $S_{\text {free }}[\mathbf{R}, \mathbf{r}]=-m \int_{0}^{t} d \tau \ddot{R} r$. The action functional $S_{F}[\mathbf{R}, \mathbf{r}]$ corresponds to the friction, and the functional $S_{N}[\mathbf{R}, \mathbf{r}]$ corresponds to the noise. The explicit expression for the friction action functional, assuming an ohmic bath, is

$$
S_{F}[\mathbf{R}, \mathbf{r}]=\eta \int_{0}^{t} d \tau \nabla w(\mathbf{r}) \cdot \dot{\mathbf{R}}
$$

The general expression for the noise functional is

$$
\begin{array}{r}
S_{N}\left[\mathbf{x}^{\prime}, \mathbf{x}^{\prime \prime}\right]=\frac{1}{2} \int_{0}^{t} \int_{0}^{t} d t_{1} d t_{2} \phi\left(t_{2}-t_{1}\right) \times \\
{\left[w\left(\mathbf{x}_{2}^{\prime \prime}-\mathbf{x}_{1}^{\prime \prime}\right)+w\left(\mathbf{x}_{2}^{\prime}-\mathbf{x}_{1}^{\prime}\right)-2 w\left(\mathbf{x}_{2}^{\prime \prime}-\mathbf{x}_{1}^{\prime}\right)\right]}
\end{array}
$$

where $\mathbf{x}_{i}$ is a short notation for $\mathbf{x}\left(t_{i}\right)$. The power spectrum of the noise $\phi(\omega)$ is the FT of the noise kernel $\phi(\tau)$. For ohmic bath

$$
\phi(\omega)=\eta \omega \hbar \operatorname{coth}\left(\frac{\hbar \omega}{2 k_{B} T}\right) \quad \text { for } \omega<1 / \tau_{c}
$$


The ZCL version for $S_{F}$ and $S_{N}$ is obtained by taking the limit $\ell \rightarrow \infty$ which is equivalent to the formal substitution $w(\mathbf{r})=-\mathbf{r}^{2} / 2$.

The power spectrum (22) of the quantum mechanical noise is the same as that of a classical white noise in the restricted frequency regime $\omega<k_{B} T / \hbar$, where $\phi(\omega)=2 \eta k_{B} T$. If the temperature is high enough, such that $1 / \tau_{c}<k_{B} T / \hbar$, then the power spectrum is essentially classical. At lower temperatures the power spectrum contains a non-trivial frequency zone $k_{B} T / \hbar<\omega<1 / \tau_{c}$, where one may use the approximation $\phi(\tau)=\hbar \eta \omega$. This component of the power spectrum does not vanish in the limit of zero temperature. It corresponds to the "zero point fluctuations" (ZPF) of the environmental modes. The temperature becomes effectively zero if $k_{B} T / \hbar<1 / t$. At intermediate temperatures, namely $1 / t<k_{B} T / \hbar<1 / \tau_{c}$, it is convenient to write the power spectrum as a sum $\phi(\omega)=\phi_{0}(\omega)+\phi_{T}(\omega)$, where $\phi_{0}(\omega)$ corresponds to the $\mathrm{ZPF}$ and $\phi_{T}(\omega)$ is the excess thermal noise:

$$
\phi_{T}(\omega)=2 \eta \frac{\hbar|\omega|}{\exp \left(\frac{\hbar|\omega|}{k_{B} T}\right)-1} \quad \text { for } \omega<1 / \tau_{c}
$$

The propagator (18) possess a Markovian property if it is legitimate to make the WNA. The actual condition for having effectively white noise will be discussed in a later section. At low temperatures it is essential to consider the non-trivial nature the quantal noise in order to obtain the proper quantum-mechanical dynamical behavior 10].

\section{Propagation in High Temperature Environment}

In the absence of noise and friction, the free-motion propagator of Wigner function is the same as in the classical limit. Namely,

$$
\mathcal{K}\left(R, P \mid R_{0}, P_{0}\right)=\mathcal{K}_{\text {free }}^{(c l)}=2 \pi \delta\left(P-P_{0}\right) \delta\left(\left(R-R_{0}\right)-\frac{P}{m} t\right)
$$

For simplicity, but without loss of generality, we shall consider whenever possible onedimensional motion. In case of the ZCL model, both $S_{F}$ and $S_{N}$ are quadratic forms, and therefore the propagator is a Gaussian kernel. It follows that the dynamics of Wigner function is purely stochastic, and it can be described by Langevin equation (11) with (2) and (22). In this sense, there are no genuine quantum mechanical effects that are associated with Brownian motion, as long as the ZCL model is used for its description. The situation is quite different in case of the DLD model. Here the propagator is non-Gaussian, and, in general, it may have non-stochastic features.

In the present section we shall restrict ourselves to the high temperature regime, where the white noise approximation (WNA), namely $\phi(\tau)=\nu \delta(\tau)$ can be applied. Whenever the WNA applies, the ZCL propagator becomes identical with the classical propagator for damped motion, namely

$$
\mathcal{K}\left(R, P \mid R_{0}, P_{0}\right)=\mathcal{K}_{\text {damped }}^{(c l)}=\text { Gaussian }
$$

In contrast, in case of the DLD model, the high temperature limit of the propagator

does not coincide with its classical limit. An explicit calculation, as in [2], gives the following expression:

$$
\mathcal{K}=W_{\hbar / \ell} \star \mathcal{K}_{\text {damped }}^{(c l)}+\mathrm{e}^{-\frac{2 \eta k_{B} T \ell^{2}}{\hbar^{2}} t}\left(1-W_{\hbar / \ell}\right) \mathcal{K}_{\text {free }}^{(c l)}
$$

This expression is valid also for the generalized DLD model provided $0<\sigma$. The precise value of the parameter $\ell$ is defined here via $w(0) \equiv \ell^{2}$. Above, $W\left(R-R^{\prime}, P-P^{\prime}\right)$ 

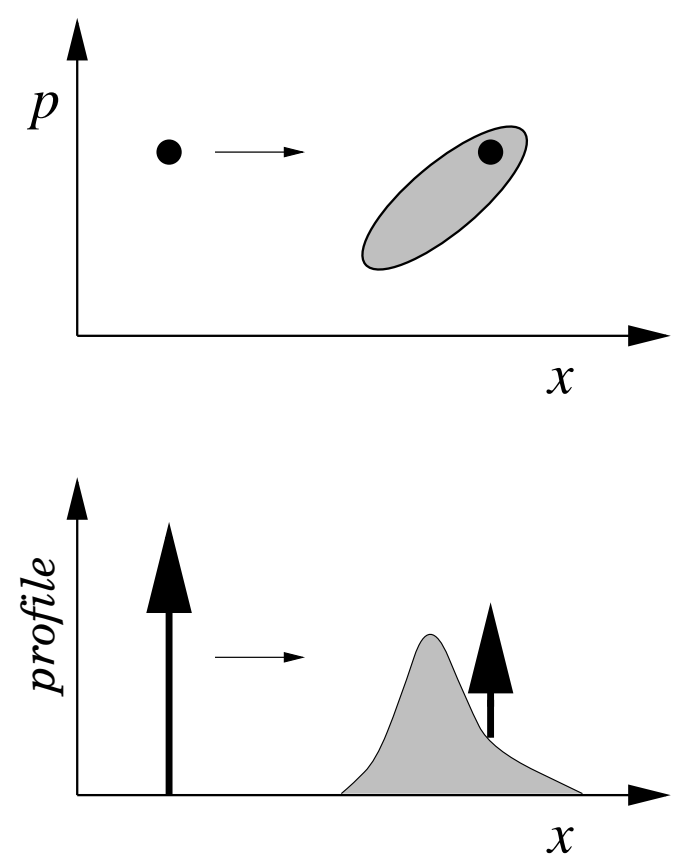

Figure 1. Upper plot: Phase space illustration for the structure of the DLD propagator. Lower plot: The projected phase-space density.

is a smooth Gaussian-like kernel that has unit-normalization. Its spread in phase space is characterized by the momentum scale $\hbar / \ell$, and by an associated spatial scale. The symbol $\star$ stands for convolution. Thus, the classical propagator is smeared on a phase-space scale that correspond to $\Delta p=\hbar / \ell$ and there is an additional un-scattered component that decay exponentially and eventually disappears. The structure of the propagator is illustrated in Fig.11. The significance of this structure will be discussed in the next section.

To write down an explicit expression for the propagator is not very useful. Rather, it is more illuminating to follow the standard procedure [3] and to write an equivalent Master equation. This is possible since at high temperatures the pathintegral expression possess a Markovian property. Namely, since both $S_{F}$ and $S_{N}$ are local functionals of the paths, it is possible to regard the finite-time propagation as the convolution of (infinitesimal) short-time kernels. The derivation of the Master equation is explained in Appendix B. The final result is

$$
\frac{\partial \rho}{\partial t}=\left[-\partial_{R} \frac{1}{m} P+\partial_{P} \frac{\eta}{m} G_{F} \star P+\nu G_{N} \star\right] \rho
$$

The friction kernel is defined as follows

$$
G_{F} \equiv \mathcal{F} \mathcal{T}\left(\frac{w^{\prime}(r)}{r}\right)=\frac{1}{\hbar / \ell} \hat{G}_{F}\left(\frac{P-P^{\prime}}{\hbar / \ell}\right)
$$

and the noise kernel is

$$
G_{N} \equiv \frac{1}{\hbar^{2}} \mathcal{F} \mathcal{T}(w(r)-w(0))=
$$




$$
\left(\frac{\ell}{\hbar}\right)^{2}\left[\frac{1}{\hbar / \ell} \hat{G}_{N}\left(\frac{P-P^{\prime}}{\hbar / \ell}\right)-\delta\left(P-P^{\prime}\right)\right]
$$

Both $\hat{G}_{F}$ and $\hat{G}_{N}$ are smooth Gaussian-like scaling functions, properly normalized to unity.

If Wigner function does not possess fine details on the momentum scale $\hbar / \ell$, then the convolution with $G_{F}$ can be replaced by multiplication with 1 , and the convolution with $G_{N}$ can be replaced by $\partial^{2} / \partial P^{2}$. These replacements are formally legitimate both in the classical limit $\hbar \rightarrow 0$, and in the ZCL limit $\ell \rightarrow \infty$. One obtains then the classical Fokker Planck equation

$$
\frac{\partial \rho}{\partial t}=\left[-\partial_{R} \frac{1}{m} P+\partial_{P} \frac{\eta}{m} P+\nu \frac{\partial^{2}}{\partial P^{2}}\right] \rho
$$

The same observation can be done by inspection of (24). Namely, if the propagator acts on a smooth Wigner-function, (no features on momentum scale $\hbar / \ell$ ), then the second term becomes vanishingly small, while the first term becomes effectively classical.

\section{Dephasing Within the Wigner Picture}

Wigner function may have some modulation on a fine scale due to an interference effect. The standard text-book example of a two slit experiment will be analyzed below, where the interference pattern has the momentum scale $\hbar / \mathbf{d}$. See Fig.2. $\mathbf{d}$ is the distance between the slits. In this section we shall explain the possible mechanisms that lead to the disappearance of such interference pattern. In view of the semiclassical point of view of the next section, we use the term "dephasing" in order to refer to this disappearance effect. In the present section, as in the previous one, we are still limiting ourselves to the high temperature regime.

In case of the ZCL model, the propagator is the same as the classical one, and therefore we may adopt a simple Langevin picture in order to analyze the dephasing process. Alternatively, we may regard the dephasing process as arising from Gaussian smearing of the interference pattern by the propagator. In case of the DLD model we should distinguish between two possible mechanisms for dephasing:

- Scattering (Perturbative) Mechanism.

- Spreading (Non-Perturbative) Mechanism.

Actually, it is better to regard them as mechanisms to maintain coherence. The first mechanism to maintain coherence is simply not to be scattered by the environment. The second mechanism to maintain coherence is not to be smeared by the propagator. The first mechanism is absent in case of the ZCL model. We shall see shortly that the first mechanism is much more effective in maintaining coherence. The notions perturbative and non-perturbative are used in order to suggest a connection with an earlier work [6]. The smearing effect can be regarded as arising from accumulation of many small-momentum scattering events. A conventional perturbative approach cannot be applied in order to analyze such dephasing process, even in the limit of very faint noise.

Having in mind the specific example of a two-slit experiment, we should distinguish between two cases. If the environment is characterized by a spatial correlation scale $\ell$ that is much larger than $\mathbf{d}$, then we can 'forget' about the DLD model and just use the ZCL model. The relevant mechanism for destruction of 


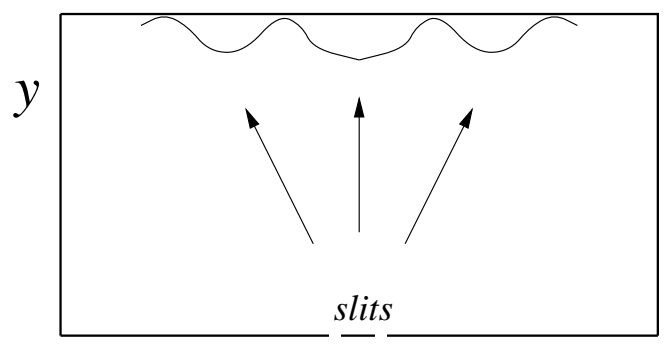

$x$

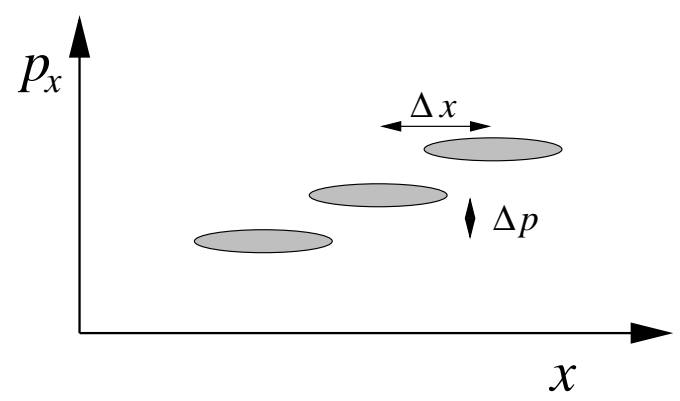

Figure 2. Upper plot: The geometry of a two-slit experiment. The propagation of the wavepacket is in the $y$ direction, and the interference pattern is resolved on the screen. Lower plot: Phase-space picture of the dynamics. Wigner function of the emerging wavepacket is projected onto the $\left(x, p_{x}\right)$ plane.

coherence in this case is the spreading mechanism. See detailed analysis in the next paragraph. If the environment characterized by a spatial correlation scale $\ell \ll \mathbf{d}$ then a totally different picture emerges. Now, Wigner function contains a modulation on a momentum scale much finer than $\hbar / \ell$. This modulation is not affected by the friction, but its intensity decays exponentially in time. This is based on inspection of either the propagator (24), or the equivalent Master equation (27). In the latter case note that the convolution with $G_{N}$ can be replaced by multiplication with $-(\ell / \hbar)^{2}$. The decay rate is

$$
\frac{1}{\tau_{\varphi}}=\frac{2 \eta k_{B} T \ell^{2}}{\hbar^{2}} \quad \text { WNA for } 0<\sigma
$$

It is a universal result for the dephasing rate due to the 'scattering mechanism', since it does not depend on details of the quantum-mechanical state involved. However, the validity of this result is restricted to the high temperature regime, where the white noise approximation (WNA) can be applied.

For completeness we turn back to the detailed analysis of dephasing due to spreading. This mechanism leads to non-universal results, since the calculation of the dephasing time depends on actual details of the interference pattern. For concreteness we consider the simplest case of a two-slit interference experiment. The wavepacket that emerges from the two slits is assumed to be a superposition $\psi(\mathbf{x})=\sum_{ \pm} \varphi(|\mathbf{x}|) \exp (i k|\mathbf{x} \mp \mathbf{d} / 2|)$, where $\hbar k$ is the momentum of the incident particle, 
and $\varphi(|\mathbf{x}|)$ is a radial envelope function. The Corresponding Wigner function is

$$
\rho(\mathbf{x}, \mathbf{p}) \approx(1+\cos (\overrightarrow{\mathbf{d}} \cdot \mathbf{p} / 4)) \times \rho_{1-\text { slit }}(\mathbf{x}, \mathbf{p})=\sum_{n=-\infty}^{\infty} \rho_{n}(\mathbf{x}, \mathbf{p})
$$

The 1-slit Wigner function is multiplied by $\cos ^{2}\left(\frac{1}{2} \mathbf{d} \cdot p_{x}\right)$, and therefore it is natural to regard the 2-slits Wigner function as composed of partial-wavepackets, each characterized by a different transverse momentum. By definition the partialwavepacket $\rho_{n}$ equals $\rho$ for $\left|p_{x}-2 \pi n / \mathbf{d}\right|<\pi / \mathbf{d}$ and equals zero otherwise. Each partial wavepacket represents the possibility that the particle, being scattered by the slits, had acquired a transverse momentum which is an integer multiplet of $\Delta p_{x}=2 \pi \hbar / \mathbf{d}$. Note that the corresponding angular separation is $\Delta p_{x} /(\hbar k)=\lambda_{B} / \mathbf{d}$, as expected. the associated spatial separation is $\Delta x=(\Delta p / m) \cdot t$ where $t=y /(\hbar k / m)$ is the time up to the screen. It is important to distinguish between the "preparation" zone $y<\mathbf{d}$ which is excluded from our considerations, and the far-field (Franhoufer) zone $\mathbf{d}^{2} / \lambda_{B} \ll y$ where $\hbar \ll \Delta x \Delta p_{x}$ and consequently the individual partial-wavepackets can be resolved. Due to the noise the interference pattern is smeared on a momentum scale $\delta p_{x}=\sqrt{\nu t}$, and on a corresponding spatial scale $\delta x=\sqrt{\nu t} \cdot t$. The interference pattern disappears once $\delta p_{x} \sim \Delta p_{x}$ or equivalently $\delta x \sim \Delta x$. This leads to the following expression for the dephasing time:

$$
\frac{1}{\tau_{\varphi}}=\frac{\eta k_{B} T}{\hbar^{2}} d^{2} \quad \text { WNA for ZCL model, two-slits }
$$

Comparing with (31) we observe that indeed the smearing mechanism is very effective in suppressing the interference pattern. The result is also non-universal since it depends on details of the interference pattern. In some other circumstances, where Wigner function is characterized by a different type of interference pattern (notably the case of "vertical" interference pattern), the dephasing time may be proportional to some fractional power of the noise intensity [6]. In general, in the full analysis of the smearing process, one should take into account the effect of friction. In the above example this effect has been neglected.

The analysis of dephasing in case of the ZCL model is easily extended to the lowtemperature regime. Langevin formalism is still applicable, provided the appropriate $\phi(\tau)$ is being used. The smearing of the interference pattern can be analyzed as in the previous paragraph. At the limit of zero temperature one should take into account the negative correlations of the noise [6]. For the ballistic-like motion that has been considered above, the smearing scale $\delta p^{2}$ is proportional, at zero temperature, to $\ln (t)$ rather than to $t$, leading to an anomalous (non-exponential) dephasing factor [6, 2]

\section{Dephasing - The Semiclassical Point of View}

There is a totally different approach to analyze dephasing, that is based on the semiclassical point of view. The advantage of the semiclassical approach is the ability to pursue the study of dephasing to the low-temperature regime as well as to circumstance in which the simple ballistic-like Brownian motion scenario is not applicable. Specific examples that will be discussed later are the transmission via either chaotic or diffusive cavity. From now on we regard the two-slit experiment as a special case of transport problem. See Fig. 3. The probability to cross the obstacle, or more generally to be transmitted via some cavity, can be written as a (double) sum 


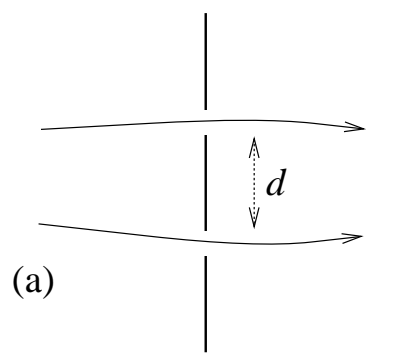

(b)

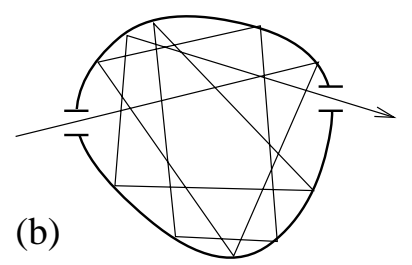

(c)

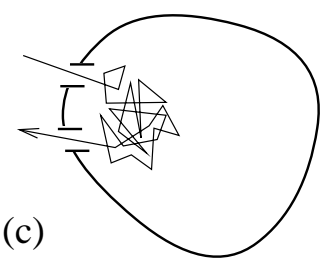

Figure 3. Various types of transport problems: (a) Ballistic transport as in the two-slit experiment; (b) Transport via a chaotic cavity; (c) Transport via diffusive cavity, as in weak localization experiments. One should consider also the case of ergodic motion via a diffusive cavity (not plotted).

over classical trajectories. Namely,

$$
\sum_{a b} A_{a} A_{b}^{*} \exp \left(i \frac{S\left[\mathbf{x}_{a}\right]-S\left[\mathbf{x}_{b}\right]}{\hbar}+i \frac{S_{F}\left[\mathbf{x}_{a}, \mathbf{x}_{b}\right]}{\hbar}-\frac{S_{N}\left[\mathbf{x}_{a}, \mathbf{x}_{b}\right]}{\hbar^{2}}\right)
$$

where $S\left[\mathbf{x}_{a}\right]$ is the classical action for the classical trajectory $\mathbf{x}_{a}$, and $A_{a}$ is the corresponding classical amplitude. Each pair $a \& b$ of classical trajectories constitutes a stationary-phase point of the exact path-integral expression. However, the notation is somewhat misleading since once the friction functional is switched on, $a$ and $b$ no longer can be considered independent indexes. In particular, strictly speaking, the diagonal terms are no longer truly diagonal. However, here comes a very important observation. In case of the DLD model, assuming that $\mathbf{x}_{a}$ and $\mathbf{x}_{b}$ are well separated with respect to the microscopic scale $\ell$, one has $S_{F}\left[\mathbf{x}_{a}, \mathbf{x}_{b}\right]=0$. Thus we conclude that friction has no effect on the interference part of the sum (34), in consistency with our discussion of the scattering-mechanism in the previous section. On the other hand, friction will have an effect on the diagonal terms of the sum, as required by the correspondence principle. It should be emphasized that in case of the ZCL model, friction may have a non-negligible effect also on the interference terms, again in consistency with our discussion of the smearing mechanism.

The suppression of an off-diagonal term in (34) is given by the dephasing factor $\exp \left(-S_{N}\left[\mathbf{x}_{a}, \mathbf{x}_{b}\right] / \hbar^{2}\right)$. Using the definitions of Appendix D it is possible to transform expression (21) into an integral over the Fourier components of the motion involved. Consequently, in most cases of interest, the dephasing factor can be cast into the form $\exp \left(-t / \tau_{\varphi}\right)$, where

$$
\frac{1}{\tau_{\varphi}}=\frac{1}{\hbar^{2}} \int_{0}^{\infty} g(k) d k \int_{0}^{\infty} \frac{d \omega}{\pi} \phi(\omega) P(k, \omega)
$$

The domain of integration is illustrated in Fig.4. The power spectrum $P(k, \omega)$ of the motion is calculated in Appendix D for ballistic, chaotic and diffusive motion. Various results that can be derived by using the above expression will be presented in later sections.

One should be very careful in the physical interpretation of $\exp \left(-t / \tau_{\varphi}\right)$. If we have two unrelated trajectories $a \& b$, and we have also static disorder, then we will have "statistical" dephasing that reflects the effect of averaging over realizations of the disorder. Consider for example a two-slit experiment: The interference pattern 
Quantal Brownian Motion -

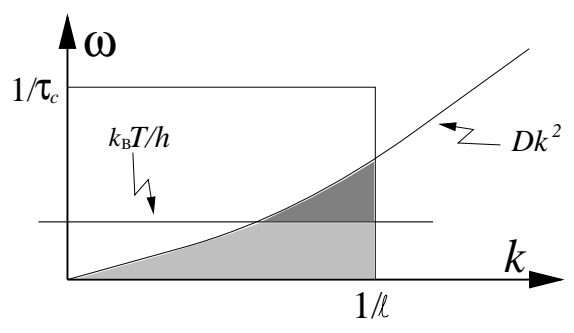

Dephasing and Dissipation

Figure 4. The $(k, \omega)$ plane. Left plot: The shaded regions indicate those environmental modes that are effective in the dephasing process. The darker region indicates a possible excess contribution due to $\mathrm{ZPF}$. The curve $D k^{2}$ illustrates the frequency-span of $P(k, \omega)$. Right plot: The power-spectrum of an ergodic motion via a diffusive cavity is concentrated under the plotted curves. See further details in Appendix D.

on the screen will be distorted for any particular realization of a static disorder, and will be washed away completely upon averaging over different realizations. This mechanism is non-effective if $a \& b$ are related by time reversal, for which static disorder gives $S_{N}\left[\mathbf{x}_{a}, \mathbf{x}_{b}\right]=0$. Still some suppression is expected also in the latter case, via the classical-like amplitudes $\left|A_{a}\right|^{2}$. In case of dynamical environment the role of elastic scattering is taken by inelastic scattering events. Therefore we have genuine dephasing, irrespective of whether $a \& b$ are related by time reversal. In some typical circumstances, the dephasing factor can be re-interpreted as giving the probability to "leave a trace" in the environment (see Appendix C) . We take now the limit of zero temperature. Assuming for simplicity that $a \& b$ are related by time reversal, it follows from the definition of the influence functional that $\exp \left(-S_{N}\left[\mathbf{x}_{a}, \mathbf{x}_{b}\right] / \hbar^{2}\right)$ can be interpreted as the probability to excite at least one of the oscillators along the way.

Up to now we have encountered, depending on the physical circumstances, two possible interpretations for the dephasing factor. The "statistical" interpretations holds in case of static disorder, while the "leaving-trace" interpretation holds in typical cases of a dynamical environment. Let us keep $T=0$ and take the limit $E \rightarrow 0$, where $E$ is the available energy of the particle. In order to make the following argumentation more illuminating, one may assume that the particle has a small but finite kinetic energy $E$, and that all the oscillators have relatively large frequencies, such that $E \ll \omega_{\alpha}$. Consequently, all the scattering events are elastic. On the other hand $1 / \tau_{\varphi}$ has a non-vanishing value, which implies that the off-diagonals terms are being suppressed. Obviously, the "leaving-trace" interpretation of the dephasing factor no longer holds. One wonders whether $\exp \left(-S_{N} / \hbar^{2}\right)$ acquires, under such circumstances, a somewhat different physical meaning, as in the case of static disorder. Maybe it gives now the probability to be scattered elastically (rather than inelastically) along the way, thus leading to dephasing of the "statistical" type. A perturbative treatment of the scattering process leads to the conclusion that the probability to be elastically scattered by a zero temperature oscillator is proportional to $c_{\alpha}^{4}$. This is because the elastic scattering off an oscillator involves a second-order process of virtual emission followed by absorption of a quanta $\hbar \omega_{\alpha}$. At the same time $S_{N}\left[\mathbf{x}_{a}, \mathbf{x}_{b}\right]$ is proportional to $c_{\alpha}^{2}$ to leading order. Therefore, $\exp \left(-S_{N} / \hbar^{2}\right)$ cannot have the desired physical significance. We therefore must conclude that something goes wrong with the present semiclassical approach once low energy particle is concerned. We shall come back to 
this point later on.

\section{Dephasing at High Temperatures}

As a first step in the application of the semiclassical approach, it is interesting to recover results (31) and (33) for the dephasing rate. These results hold at high temperatures, such that the WNA is applicable. The noise functional in such circumstances takes the following simple form

$$
S_{N}[\mathbf{r}]=2 \eta k_{B} T \int_{0}^{t}\left[w(0)-w\left(\mathbf{r}\left(t^{\prime}\right)\right)\right] d t^{\prime}
$$

where $\mathbf{r}=\mathbf{x}_{a}-\mathbf{x}_{b}$. If the two trajectories are well separated with respect to the microscopic scale $\ell$, then one indeed recovers the universal result (31). Recall that $w(0) \equiv \ell^{2}$. In the other extreme limit $\ell \rightarrow \infty$, that corresponds to the ZCL model, one obtains

$$
S_{N}[\mathbf{r}]=2 \eta k_{B} T \int_{0}^{t} \mathbf{r}\left(t^{\prime}\right)^{2} d t^{\prime} \equiv 2 \eta k_{B} T r_{\perp}^{2} \cdot t
$$

In case of the two-slit experiment $\mathbf{r} \sim \mathbf{d}$, and one recovers (33). Obviously, it is not a universal result. For example, if the separation between the two trajectories grows linearly, then the dephasing time will be proportional to $1 / T^{1 / 3}$ rather than to $1 / T$.

The generalized DLD model with $-2<\sigma<0$, requires special care since $w(0)$ diverges. We are still considering high temperatures, such that the WNA is applicable. Using the notations of Appendix D expression (36) can be cast into the form

$$
S_{N}[\mathbf{r}]=\nu t \int w(\mathbf{r}) P(\mathbf{r}) d \mathbf{r}=\nu t \int_{0}^{\infty} g(k) d k P(k)
$$

The latter expression converges for any $-2<\sigma$. In the ZCL limit, where $\ell$ is much larger than the average transverse distance between the trajectories, one may use the small- $k$ approximation of Appendix D, and then (37) is recovered. In the DLD case, where $\ell$ is a very small scale, the integration should be split into the domains $k<k_{\perp}$ and $k>k_{\perp}$. The wavenumber $k_{\perp} \equiv 1 / r_{\perp}$ is associate with the transverse distance $r_{\perp}$ between the two trajectories. Namely,

$$
r_{\perp} \approx\left\{\begin{array}{ccc}
\mathbf{d} & \text { for ballistic motion } & t<\tau_{\text {bal }} \\
\sqrt{D t} & \text { for diffusive motion } & \tau_{\text {bal }}<t<\tau_{\text {erg }} \\
L & \text { for ergodic motion } & \tau_{\text {erg }}<t
\end{array}\right.
$$

Note that ergodic motion refers to either chaotic or diffusive trajectories with $\tau_{\operatorname{erg}}<t$. Again we use the notations of Appendix D. For $0<\sigma$ the integration is dominated by the upper cutoff $k \sim 1 / \ell$. One recovers then the standard result (31). On the other hand, for $-2<\sigma<0$, the integration is dominated by the $k \sim k_{\perp}$ modes. The wavelength of these modes is like the transverse distance between the trajectories. One obtains then

$$
S_{N}[\mathbf{r}]=2 \eta k_{B} T \ell^{2}\left(\frac{\ell}{r_{\perp}}\right)^{\sigma} \cdot t \quad \text { WNA for }-2<\sigma<0
$$

For non-ergodic diffusive motion $r_{\perp}$ depends on $t$ and therefore the dephasing factor is of the type $\exp \left(-\left(t / \tau_{\varphi}\right)^{(2-\sigma) / 2}\right)$. In all other cases we have a simple exponential dephasing factor.

Obviously (38) is a special case of the general formula (35). Note that (35) should be multiplied by $\hbar^{2} t$ in order to become a proper expression for $S_{N}$. By comparing (35) 
to (38) one can reveal the actual condition for the validity of the WNA. Within the interval $0<k<1 / \ell$, the power spectrum of the motion occupies a frequency range $\omega<\omega_{c l}$, where the effective cutoff is

$$
\omega_{c l}=\left\{\begin{array}{cl}
v / \ell & \text { if } k=1 / \ell \text { is located within the ballistic regime } \\
D / \ell^{2} & \text { if } k=1 / \ell \text { is located within the diffusive regime }
\end{array}\right.
$$

As long as $\omega_{c l}<k_{B} T / \hbar$ one can use the WNA in estimating the integral in (35). One observes that under such circumstances (35) reduces, after multiplication by $\hbar^{2} t$, to (38).

\section{Dephasing at Zero Temperatures}

As the temperature becomes low, such that $k_{B} T / \hbar<\omega_{c l}$, the dephasing rate becomes larger than the value which is predicted by the WNA. This is due to the "zero point fluctuations" (ZPF) in the frequency zone $k_{B} T / \hbar<\omega$. The temperature becomes effectively zero if $k_{B} T / \hbar<1 / t$. Time longer than $t$ is required in order to resolve such low temperatures. One can use then the zero temperature limit of $\phi(\tau)$ for which $\phi(\omega)=\hbar \eta \omega$. This power spectrum corresponds to the ZPF of the environmental modes.

For either ballistic or chaotic motion, The integral (35) is dominated by $(k, \omega)$ modes that are concentrated below the curve $\omega=v k$. One obtains

$$
\frac{1}{\tau_{\varphi}} \approx \frac{C}{(1+\sigma) \pi} \times \frac{1}{\hbar} \eta \ell v \quad \text { for ballistic or chaotic motion }
$$

Similarly, for diffusive motion the integral is dominated by $(k, \omega)$ modes that are concentrated below the curve $\omega=D k^{2}$. One obtains

$$
\frac{1}{\tau_{\varphi}}=\frac{C}{(2+\sigma) \pi} \ln \left(1+\left(\frac{\ell v}{D}\right)^{4}\right) \times \frac{1}{\hbar} \eta D \quad \text { for diffusive motion (43) }
$$

In both cases most of the contribution comes from modes with large wavenumber, namely $k \sim 1 / \ell$.

The validity of the present semiclassical approach, which is based on the stationary-phase approximation is limited. Common wisdom [11] is that the applicability of the semiclassical approach is restricted to circumstances such that the energy transfer between the particle and the environment is much smaller than the particle's available energy. Technically, it is equivalent to the assumption of uncoupled wave-equations [12]. The coupled wave-equations for the particle-oscillators system can be uncoupled provided certain conditions are satisfied. Then we can treat the particle as moving with constant velocity $v$ and solve for the oscillators. It turns out that this reduction requires the assumption of small energy transfer. Therefore, one should anticipate problems once oscillators with $\omega_{\alpha}$ larger than $E$ are involved. In the latter case, there is no justification to think of the particle as decoupled from the bath, moving with some constant velocity, capable of exciting oscillators along the way. Therefore the corresponding factor $\exp \left(-S_{N} / \hbar^{2}\right)$ loses its physical significance. From the above considerations it follows that a reasonable condition for the validity of our semiclassical approach is

$$
\hbar \omega_{c l}<E \quad \text { definition of large energy }
$$

On physical grounds this is the condition for being able to leave a trace "along the way". The semiclassical significance of this condition can be further illuminated. For ballistic-like motion it is equivalent to the condition of small momentum transfer 
$\hbar / \ell<p$. This is precisely the condition for the applicability of the semiclassical methods for the scattering of the particle by the oscillators. More generally, one can define a quantum mechanical version of $P(k, \omega)$ as the FT of the correlator of the Heisenberg-picture operator $\exp (i k \mathbf{x}(t))$. The quantal $P(k, \omega)$ coincides with the classical $P(k, \omega)$ only for $\omega<E / \hbar$. Larger classical frequencies are not supported by the effectively banded energy spectrum. Our practical conclusion is that the contribution of ZPF should be included if non-thermal motion with large energy is concerned. It should be excluded in case of thermal motion with $E \sim k_{B} T$.

\section{Dephasing due to Thermal Noise}

The possible contribution of ZPF to the dephasing has been discussed in the previous section. Now we are interested in the the thermal noise contribution (TNC). The TNC can be calculated using (35) with $\phi(\omega)$ replaced by $\phi_{T}(\omega)$. See (23) for the definition of the latter. As the temperatures is being raised various regimes are encountered:

$$
\begin{array}{lll}
\text { Extremely Low Temperatures } & \Leftrightarrow \quad 1 / t<k_{B} T / \hbar<1 / \tau_{\perp} \\
\text { Low Temperatures } & \Leftrightarrow \quad 1 / \tau_{\perp}<k_{B} T / \hbar<\omega_{c l} \\
\text { High Temperatures } & \Leftrightarrow \quad \omega_{c l}<k_{B} T / \hbar
\end{array}
$$

The time scale $\tau_{\perp}$ corresponds to the transverse distance $r_{\perp}$ of (39). It equals $\mathbf{d} / v$ for ballistic motion, and $\tau_{\text {erg }}$ for ergodic motion. In case of non-ergodic diffusive motion, where $\tau_{\perp}=t$, the extremely low temperature regime is absent. At high temperatures we can use the WNA as discussed in previous sections. The low and the extremely low temperature regimes are discussed in the next two paragraphs.

When considering extremely low temperatures it is useful to define the critical exponent $\sigma_{c}=1$ for ballistic motion, and $\sigma_{c}=2$ for diffusive motion. If $\sigma<\sigma_{c}$ the integration in (35) is dominated by $k \sim k_{\perp}$ and one obtains the result

$$
\frac{1}{\tau_{\varphi}}=C^{\prime} \frac{2 \eta k_{B} T}{\hbar^{2}} \ell^{2} \times\left(\frac{\ell}{r_{\perp}}\right)^{\sigma} \times\left(\frac{k_{B} T / \hbar}{1 / \tau_{\perp}}\right)
$$

For $\sigma_{c}<\sigma$ the integration is dominated by $k \sim 1 / \ell$ and one obtains the result

$$
\frac{1}{\tau_{\varphi}}=C^{\prime} \frac{2 \eta k_{B} T}{\hbar^{2}} \ell^{2} \times\left(\frac{k_{B} T / \hbar}{\omega_{c l}}\right)
$$

In the formulas above $C^{\prime}$ is a numerical factor of order unity. Note that in both cases, disregarding possible contribution of ZPF, the dephasing rate is proportional to $T^{2}$. This should be contrasted with the high temperature behavior where, disregarding the special case of non-ergodic diffusive motion, the dephasing rate is proportional to $T$.

At low (but not extremely low) temperatures it is useful to define a temperature dependent wavenumber as follows:

$$
k_{T}= \begin{cases}\left(k_{B} T / \hbar v\right) & \text { in the ballistic regime } \\ \left(k_{B} T / \hbar D\right)^{1 / 2} & \text { in the diffusive regime }\end{cases}
$$

For $\sigma<0$ the integration in (35) is dominated by $k \sim k_{\perp}$, and one can use the WNA result (40). Thus, for $\sigma<0$, in the absence of ZPF contribution, we can trust the WNA at both high and low temperatures, and an actual crossover is expected only when extremely low temperatures are involved. For $0<\sigma<\sigma_{c}$ the integration is dominated by $k \sim k_{T}$, and one obtains the result

$$
\frac{1}{\tau_{\varphi}}=C^{\prime} \frac{2 \eta k_{B} T}{\hbar^{2}} \ell^{2} \times\left(\ell k_{T}\right)^{\sigma}
$$


If $\sigma_{c}<\sigma$ the integration is dominated by $k \sim 1 / \ell$ and one obtains again (47). Thus, for $0<\sigma<\sigma_{c}$, there is a non-trivial low-temperature regime where the TNC to the dephasing rate is proportional to a non-universal power of $T$. Below this intermediate temperature-regime the TNC is proportional to $T^{2}$. Above this intermediate temperature-regime we can trust the WNA and the dephasing rate is proportional to $T$.

\section{Manifestation of Effective Static Disorder}

We shall consider in this section quantal Brownian motion in $2<d$ dimensions, that is described by the the DLD model with short range spatial correlations. For concreteness let us assume that the fluctuations of the effective stochastic potential satisfy:

$$
\left\langle\mathcal{U}\left(\mathbf{x}^{\prime \prime}, t^{\prime \prime}\right) \mathcal{U}\left(\mathbf{x}^{\prime}, t^{\prime}\right)\right\rangle=\phi\left(t^{\prime \prime}-t^{\prime}\right) \cdot \ell^{2} \exp \left(-\frac{1}{2}\left(\frac{\mathbf{x}^{\prime \prime}-\mathbf{x}^{\prime}}{\ell}\right)^{2}\right)
$$

If we are interested in the dynamics over a finite time interval $t$, then obviously all the Fourier components in the frequency regime $|\omega|<1 / t$ will have the same effect as static disorder. Let us denote by $\overline{\mathcal{U}}(\mathbf{x}, t)$ the "static" component of the effective stochastic potential. The variance of $\overline{\mathcal{U}}$ is determined by the product $\phi(\omega=0) \cdot(1 / t)$. Recall that $\phi(\omega=0) \equiv \nu$. Consequently we have

$$
\left\langle\overline{\mathcal{U}}\left(\mathbf{x}^{\prime \prime}, t^{\prime \prime}\right) \overline{\mathcal{U}}\left(\mathbf{x}^{\prime}, t^{\prime}\right)\right\rangle=W^{2} \exp \left(-\frac{1}{2}\left(\frac{\mathbf{x}^{\prime \prime}-\mathbf{x}^{\prime}}{\ell}\right)^{2}\right)
$$

where $W^{2}=\nu \ell^{2} / t$. We would like to find out whether this effective static disorder will manifest itself. Similar question has been raised in 13]. One should not take for granted that the effect of low-frequency fluctuations is completely masked by the the incoherent effect of the high frequency modes.

The first obvious step is to calculate the "statistical" dephasing rate due to the presence of the (effective) disorder. We can use (35) with the formal substitution $\phi(\tau)=W^{2} / \ell^{2}$, thus obtaining

$$
\frac{1}{\tau_{\varphi}^{0}}=\frac{1}{\hbar^{2}} \frac{W^{2}}{\ell^{2}} \int_{0}^{\infty} g(k) d k P(k, \omega=0)
$$

For ballistic-like motion one obtains

$$
\frac{1}{\tau_{\varphi}^{0}}=\frac{1}{\hbar^{2}} W^{2} \frac{\ell}{v}=\left(\frac{\nu \ell^{3}}{\hbar^{2} v}\right) \cdot \frac{1}{t}
$$

In order to have a non-vanishing effect we should have $\tau_{\varphi}^{0} \ll t$. This condition can be cast into the form $\xi \ll v t$ where $\xi=(\hbar v)^{2} /\left(\ell W^{2}\right)$ is the mean free path. Still another way to express this condition is $\tau_{\varphi}^{\mathrm{WNA}} \ll \ell / v$, where $\tau_{\varphi}^{\mathrm{WNA}}$ is given by (31). On the over hand, the actual dephasing time should satisfy $\ell / v<\tau_{\varphi}$, else coherent effects due to the scattering by the (effective) disordered potential will not manifest themselves. Thus, we come to the conclusion that the following condition should be satisfied in order to have manifestation of coherent effects due to scattering by the effective static disorder:

$$
\tau_{\varphi}^{\mathrm{WNA}} \ll \frac{\ell}{v} \ll \tau_{\varphi}
$$

Obviously, this condition can be satisfied only at the low temperature regime. 


\section{Dephasing versus Dissipation, Concluding Remarks}

It is quite striking that the friction coefficient $\eta$ is not affected by quantum mechanical effects. Having the Boltzmann picture in mind it is not anticipated that all the quantum mechanical scattering events will conspire to give the classical result. Still, this conclusion follows from the FV-formalism quite easily. In order to have a clear physical picture, let us consider the time evolution of a Gaussian wavepacket using either the propagator (26) or the equivalent Master equation (27). Clearly, the dissipation rate $d E / d t$ is the same as in the classical picture, with small transient corrections. Thus, also in the quantum-mechanical picture, the damping process is characterized by the time constant $\tau_{\eta}=(\eta / m)^{-1}$.

Consider a ballistic-like motion with non-thermal energy, such that $\hbar / \ell \ll p$. Consequently the equivalent condition (44) is satisfied, and we can trust semiclassical considerations as far as dephasing is concerned. We can cast the universal WNA result (31) and the ZPF result (42) into the form

$$
\frac{1 / \tau_{\varphi}}{1 / \tau_{\eta}} \approx\left\{\begin{array}{cc}
\left(\ell / \lambda_{T}\right)^{2} & \text { high-T } \\
\left(\ell / \lambda_{B}\right) & \text { low-T }
\end{array}\right.
$$

where $\lambda_{T}$ is the thermal wavelength, and $\lambda_{B}$ is the De-Broglie wavelength. We see that for the above non-thermal motion the dephasing time is always shorter than the damping time. The dephasing rate is linear in $T$ at high temperature, and saturates at low temperatures.

For thermal motion the latter statement is no-longer true. At the low temperature regime the ZPF contribution should be excluded, and therefore the dephasing rate goes to zero, while the damping time remains finite. One should distinguish between various physical circumstances. At extremely low temperatures, assuming ergodic motion, the dephasing rate is proportional to $T^{2}$. At higher temperatures we can trust the WNA provided $\sigma<0$. Otherwise, there is an intermediate low-temperature regime where the dephasing rate is proportional to a non-trivial power of $T$.

In this paper we have introduced a systematic derivation of a general formula for the dephasing rate, Eq.(35), that holds in all physical circumstances, including the ZCL limit. Expressions similar to Eq.(35), that incorporate integration over the $(k, \omega)$ environmental modes, are encountered frequently in the literature, starting from the well known work in 14. Whenever diffusive motion of electrons is concerned (see Appendix E), our expressions agree with well know results 77. However, most publications avoid a straightforward application of the FV formalism, and introduce in some stage heuristic considerations in order to obtain a convergent result. There is a "zoo" of cutoffs that are introduced in performing the $(k, \omega)$ integration, some of them are questionable. For example, it is customary to take $1 / \tau_{\varphi}$ itself as a lower cutoff for the $\omega$ integration. The upper cutoff is sometimes $k_{B} T / \hbar$, sometimes Fermi energy, sometimes the kinetic energy that corresponds to the drift velocity, sometimes the inelastic scattering rate, and so on. The role of Fermi statistics in the determination of the various cutoffs is usually left unclear. There are similar ambiguities in the determination of the the proper cutoffs for the $k$ integration. Our derivation has led to a proper definition for the power-spectrum of the motion, and has made it unnecessary to introduce ad-hoc cutoffs into the calculations. Furthermore, in the analysis of dephasing at the limit of zero temperature, we were successful in reducing our considerations to the level of "one particle physics", thus avoiding a complicated discussion of the role played by Pauli exclusion principle. 


\section{Acknowledgments}

I thank Uzy Smilansky and Yoseph Imry for useful discussions. Some of the motivation for this work has come following suggestions by Yuval Gefen. This research was supported by the Minerva Center for Nonlinear Physics of Complex systems.

\section{Appendix A. Useful Identity}

We first cite the well known identity

$$
\frac{1}{\sqrt{2 \pi \delta t}} \exp \left(+\frac{i}{2 \delta t}\left(x-x_{0}\right)^{2}\right)=\left[1+\frac{i}{2} \delta t \partial_{x}^{2}+\mathcal{O}\left(\delta t^{2}\right)\right] \delta\left(x-x_{0}\right)
$$

Both sides of this identity should be interpreted as kernels of operators. When applied to wave-functions, the left hand side corresponds to the free-motion propagator, and the second term in the right hand side corresponds to the free-motion Hamiltonian. We shall explain now how to derive the following related identity:

$$
\begin{aligned}
& \frac{1}{2 \pi \delta t} \exp \left(\frac{i}{\delta t}\left(R-R_{0}\right)\left(r-r_{0}\right)-i \eta \cdot\left(R-R_{0}\right)\right)= \\
& {\left[1+i \delta t \partial_{R} \partial_{r}+\eta \delta t \partial_{r}+\mathcal{O}\left(\delta t^{2}\right)\right] \delta\left(R-R_{0}\right) \delta\left(r-r_{0}\right)}
\end{aligned}
$$

Both sides of the latter identity should be interpreted as kernels of operators that operate on phase-space functions. We start the derivation by writing an equality that follows from the first identity via simple replacements:

$$
\left.\frac{1}{\sqrt{2 \pi \delta t}} \exp \left( \pm \frac{i}{2 \delta t}\left(x_{ \pm} \mp \frac{1}{2} \eta \delta t\right)\right)^{2}\right)=\left[1+\frac{i}{2} \delta t \partial_{ \pm}^{2}+\mathcal{O}\left(\delta t^{2}\right)\right] \delta\left(x_{ \pm} \mp \frac{1}{2} \eta \delta t\right)
$$

Upon multiplication it follows that

$$
\begin{aligned}
& \frac{1}{\sqrt{2 \pi \delta t}} \exp \left( \pm \frac{i}{2 \delta t}\left(x_{+}^{2}-x_{-}^{2}\right)-i \frac{1}{2} \eta\left(x_{+}+x_{-}\right)\right)= \\
& {\left[1+\frac{i}{2} \delta t \partial_{+}^{2}\right]\left(\delta\left(x_{+}\right)-\frac{1}{2} \eta \delta t \delta^{\prime}\left(x_{+}\right)\right) \cdot\left[1-\frac{i}{2} \delta t \partial_{-}^{2}\right]\left(\delta\left(x_{-}\right)+\frac{1}{2} \eta \delta t \delta^{\prime}\left(x_{+}\right)\right)}
\end{aligned}
$$

Simplification of the right hand side gives

$$
=\left[1+\frac{i}{2} \delta t\left(\partial_{+}^{2}-\partial_{-}^{2}\right)+\frac{1}{2} \eta \delta t\left(\partial_{+}-\partial_{-}\right)+\mathcal{O}\left(\delta t^{2}\right)\right] \delta\left(x_{+}\right) \delta\left(x_{-}\right)
$$

The replacements $x_{+} \rightarrow\left(x^{\prime \prime}-x_{0}^{\prime \prime}\right)$ and $x_{-} \rightarrow\left(x^{\prime}-x_{0}^{\prime}\right)$, followed by the transformation to the variables $R=\left(x^{\prime \prime}+x^{\prime}\right) / 2$ and $r=\left(x^{\prime \prime}-x^{\prime}\right)$, gives the desired result. Note that the derivation holds also in the case where $\eta$ is replaced by some function of $r$.

\section{Appendix B. Derivation of the Master Equation}

Wigner function $\rho(R, P)$ is the Fourier transform of the reduced probability density matrix $\rho(R, r)$ in the variable $r \leadsto P$. The path integral expression for the kernel $\mathcal{K}\left(R, r \mid R_{0}, r_{0}\right)$ is the type $\iint \mathcal{D} R \mathcal{D} r$ with an obvious endpoint conditions, and its integrand is essentially the same as in (19). See [2] for more details. The infinitesimaltime kernel equals simply to the integrand of the path integral expression, namely

$$
\begin{aligned}
& \mathcal{K}_{\delta t}=\exp \left(\frac{i}{\delta t} \frac{m}{\hbar}\left(R-R_{0}\right)\left(r-r_{0}\right)+i \frac{\eta}{m} w^{\prime}(r) \cdot\left(R-R_{0}\right)-\delta t \frac{\nu}{\hbar^{2}}(w(0)-w(r))\right)= \\
& {\left[1+\delta t\left(i \frac{\hbar}{m} \partial_{R} \partial_{r}-\frac{\eta}{m} w^{\prime}(r) \partial_{r}-\frac{\nu}{\hbar^{2}}(w(0)-w(r))\right)+\mathcal{O}\left(\delta t^{2}\right)\right] \delta\left(R-R_{0}\right) \delta\left(r-r_{0}\right)}
\end{aligned}
$$


where the second line is obtained by employing the identity of Appendix A. Thus, to leading order, the kernel $\mathcal{K}_{\delta t}$ has the same effect as operating with a differential operator of the type $1+\delta t \mathcal{L}$. Consequently, we find that $\rho(R, r)$ satisfies a Master equation of the form $\partial \rho / \partial t=\mathcal{L} \rho$. This Master equation is easily transformed into the Wigner representation, where any derivative $\partial_{r}$ is replaced by multiplication with $i P / \hbar$. Similarly, any multiplication by a (real symmetric) function $\tilde{G}(r)$ is transformed into a convolution with a (real symmetric) kernel $G\left(P-P^{\prime}\right)$. For the convolution $\int G\left(P-P^{\prime}\right) \rho\left(P^{\prime}\right) d P^{\prime}$ we use the notation $G \star \rho$. A kernel $G\left(P-P^{\prime}\right)$ and a function $\tilde{G}(r)$ are related by a Fourier transform with the convention

$$
G\left(P-P^{\prime}\right)=\frac{1}{2 \pi \hbar} \int_{-\infty}^{\infty} G(r) \cos \left(\frac{P-P^{\prime}}{\hbar} r\right) d r
$$

In the above expression we have modified the standard FT convention, by including the factor $1 /(2 \pi \hbar)$. This has been done in order to have properly normalized kernels, with the measure $d P$ rather than $d P /(2 \pi \hbar)$. Note also that the FT of $w^{\prime}(r)$ equals to the $i \hbar \partial_{P}$ derivative of the kernel $G_{F}$ that corresponds to the real symmetric function $w^{\prime}(r) / r$.

\section{Appendix C. Dephasing and Inelastic Scattering}

Consider the interference contribution of two trajectories $a \& b$ that are related by time reversal. Assuming short range interaction with the environmental modes we can prove that dephasing is related to the probability for leaving a trace in the environment. This statement is true at any temperature. "Short range interaction" means that $0<\sigma$ and that $\ell$ is a small scale. "Leaving a trace" means that at least one of the oscillators has changed its quantum-mechanical state. It follows that under such circumstances dephasing-rate is equal to the inelastic scattering rate.

Recall the definition of the influence functional. For simplicity we consider first a zero-temperature bath, meaning that all the bath-oscillators are initially in the ground state. These oscillators are driven by the motion of the particle. The excited states of the bath will be denoted by $\left|\left\{n_{\alpha}\right\}\right\rangle$. The evolution operator of a driven oscillator will be denoted by $U_{\alpha}[x]$.

$$
F\left[\mathbf{x}_{a}, \mathbf{x}_{b}\right] \equiv \sum_{\left\{n_{\alpha}\right\}}\left\langle\left\{n_{\alpha}\right\}\left|\prod_{\alpha} U_{\alpha}\left[\mathbf{x}_{b}\right]\right|\{0\}\right\rangle\left\langle\left\{n_{\alpha}\right\}\left|\prod_{\alpha} U_{\alpha}\left[\mathbf{x}_{a}\right]\right|\{0\}\right\rangle^{\star}
$$

If $a \& b$ are related by time reversal, then one can consider only those oscillators that are located "along the loop". Each oscillator along the loop is characterized by its natural frequency $\omega_{\alpha}$ and by the time $t_{\alpha}$ at which $x_{a}(t) \approx x_{\alpha}$. Consequently we have the following expression for the influence functional:

$$
F\left[\mathbf{x}_{a}, \mathbf{x}_{b}\right]=\sum_{\left\{n_{\alpha}\right\}} \mathrm{e}^{-i \sum_{\alpha} n_{\alpha} \omega_{\alpha} \cdot\left(2 t_{\alpha}-t\right)} P\left(\left\{n_{\alpha}\right\} \mid\{0\}\right)
$$

where the excitation probability is $P\left(\left\{n_{\alpha}\right\} \mid\{0\}\right) \equiv \prod\left|\left\langle n_{\alpha}\left|U_{\alpha}\left[\mathbf{x}_{a}\right]\right| 0\right\rangle\right|^{2}$. We would like to argue that $F\left[\mathbf{x}_{a}, \mathbf{x}_{b}\right]=P(\{0\} \mid\{0\})$. Indeed, the summation in (C.1) contains one-oscillator excitations for which $\sum n_{\alpha}=1$, two-oscillator excitations for which $\sum n_{\alpha}=2$ and so on. Let us consider all the one-oscillator excitations that involves $\omega_{\alpha}$ in the range $[\omega, \omega+\delta \omega]$. Each of these excitations contributes the same $P\left(\left\{n_{\alpha}\right\} \mid\{0\}\right)$, but with a different phase factor $\exp \left(i 2 \omega_{\alpha} t_{\alpha}\right)$. By construction of the DLD model 
(Eq.12), the summation over the phase factors will lead to a zero contribution. The meaning of "zero contribution" is as follows: either we average over realizations of $x_{\alpha}$, or else we recall that the influence functional appears inside a path-integral expression. The summation over paths will have the same effect like an averaging procedure, and therefore we will have indeed a "zero contribution" in the mathematically-generalized sense. Similar argumentation applies to other subsets of excitations. If the bath is initially in thermal equilibrium, rather than in zero temperature, than the relation $F\left[\mathbf{x}_{a}, \mathbf{x}_{b}\right]=P(\{0\} \mid\{0\})$ can be generalized into

$$
F\left[\mathbf{x}_{a}, \mathbf{x}_{b}\right]=\exp \left(-S_{N}\left[\mathbf{x}_{a}, \mathbf{x}_{b}\right] / \hbar^{2}\right)=P(\text { leaving no trace })
$$

In order to prove the latter statement one should use the same procedure as above with $|\{0\}\rangle$ replaced by some arbitrary initial preparation $\left|\left\{n_{\alpha}^{0}\right\}\right\rangle$. Then the result should be thermally averaged.

\section{Appendix D. Statistical Characterization of the Trajectories}

Having a pair $a \& b$ of trajectories, we can define the function

$$
P_{a b}(\mathbf{r}, \tau)=\left\langle\delta\left(\mathbf{r}-\left(\mathbf{x}_{a}\left(t^{\prime}+\tau\right)-\mathbf{x}_{b}\left(t^{\prime}\right)\right)\right)\right\rangle
$$

where the average is over $t^{\prime}$ within the time interval $[0, t]$ which is considered. We shall use the notations $P_{a a}=P_{\|}$and $P_{a b}=P_{\perp}$ for $a \neq b$. It is also useful to define the functions

$$
\begin{aligned}
& P(\mathbf{r}, \tau) \equiv P_{\|}(\mathbf{r}, \tau)-P_{\perp}(\mathbf{r}, \tau) \\
& P(\mathbf{r}) \equiv P(\mathbf{r}, 0) .
\end{aligned}
$$

The Fourier transform of $P(\mathbf{r})$ will be denoted by $P(\mathbf{k})$, and the double Fourier transform of $P(\mathbf{r}, \tau)$ will be denoted by $P(\mathbf{k}, \omega)$. The above functions all appear within integrals where "isotropic" integration over $\mathbf{r}$ or $\mathbf{k}$ is being performed. Therefore it is convenient to average all these functions over all orientations, and so to have functions that depend on either $r=|\mathbf{r}|$ or $k=|\mathbf{k}|$ respectively. A useful notation is $\operatorname{Cos}(\mathbf{r}) \equiv\langle\cos (\boldsymbol{\Omega} \cdot \mathbf{r})\rangle$, where the average is over the orientation of the unit vector $\boldsymbol{\Omega}$. The function $\operatorname{Cos}(\mathbf{r})$ depends only on $|\mathbf{r}|$. It equals $\cos (r)$ in 1-D, regular Bessel function $\mathcal{J}_{0}(r)$ in $2-\mathrm{D}$, and $\operatorname{sinc}(r)$ in $3-\mathrm{D}$.

We shall distinguish now between ballistic trajectories as in Fig.3(a), chaotic trajectories as in Fig.3(b), and diffusive trajectories as in Fig.3(c). We can also add to this list the case of diffusive trajectories that cover ergodically the whole available space. In case of a ballistic trajectory $P_{\|}(\mathbf{r}, \tau)=\delta(\mathbf{r}-\overline{\mathbf{r}})$, leading to $P_{\|}(\mathbf{k}, \tau)=\operatorname{Cos}(k \bar{r})$, where $\bar{r}=v \tau$. Similar expression hold for $P_{\perp}$, where $\bar{r} \approx\left(\mathbf{d}^{2}+(v \tau)^{2}\right)^{-1 / 2}$, and $\mathbf{d}$ is the transverse distance between the slits. For a chaotic trajectory $P_{\|}(r, \tau)$ starts as in the ballistic case, while $P_{\perp}(r, \tau)$ is uniform in $\mathbf{r}$. The ergodic time is given essentially by the ballistic time, namely $\tau_{\text {erg }}=\tau_{\text {bal }}=L / v$, where $L$ is the linear dimension of the cavity. For $\tau_{\text {erg }}<\tau$ both $P_{\|}(r, \tau)$ and $P_{\perp}(r, \tau)$ become uniform in $\mathbf{r}$. For diffusive motion $P_{\|}(\mathbf{r}, \tau)$ is as for the ballistic case as long as $\tau<\tau_{\text {bal }}$ where $\tau_{\text {bal }}=D / v^{2}$. On larger times it becomes a Gaussian, and consequently $P_{\|}(\mathbf{k}, \tau)=\exp \left(-D k^{2} \tau\right)$, where $D$ is the diffusion coefficient. The latter expression as well as the approximation $P_{\perp}(\mathbf{r}, \tau) \approx P_{\|}(\mathbf{r}, t)$ hold for non-ergodic diffusive motion. After the ergodic time $\tau_{\text {erg }}=L^{2} / D$, both $P_{\|}(r, \tau)$ and $P_{\perp}(r, \tau)$ become uniform in $\mathbf{r}$.

In the following paragraphs we discuss the various $k$ regimes of the function $P(k, \omega)$. We shall distinguish between the small- $k$ regime and the large- $k$ regime. In 
case of diffusive trajectories the large- $k$ regime will be further divided into a ballistic regime $(v / D<k)$ and a diffusive regime $(k<v / D)$, where $v / D$ is the inverse of the mean free path. We turn first to define the notions of small and large $k$. The transverse distance between two trajectories has a distribution $P_{\perp}(r)$, and we can define a typical value $r_{\perp}=\left(\left\langle\mathbf{r}^{2}\right\rangle\right)^{1 / 2}$. See Eq.(39). The associated wavenumber is $k_{\perp} \equiv 1 / r_{\perp}$. Large $k$ means from now on $k_{\perp} \ll k$. For large $k$ we may use the approximation

$$
P(k, \omega) \approx P_{\|}(k, \omega) \quad \text { for large } k \text { (meaning } k_{\perp} \ll k \text { ) }
$$

For either ballistic, diffusive or chaotic trajectories we have

$$
P(k, \omega) \approx \frac{1}{v k} \hat{B}\left(\frac{\omega}{v k}\right) \quad \text { in the ballistic regime }
$$

where $\hat{B}$ is the FT of the $\operatorname{Cos}$ function. This rectangular-like scaling-function has a unit width and a unit normalization. In case of diffusive motion we also have

$$
P(k, \omega) \approx \frac{2 D k^{2}}{\left(D k^{2}\right)^{2}+\omega^{2}} \quad \text { in the diffusive regime }
$$

Diffusive regime means here any large $k$ that satisfies $k<v / D$. The validity of the latter approximation is further restricted by the condition $\omega<v^{2} / D$. The collision frequency $\omega \sim v^{2} / D$ should be used as a cutoff to the slow $1 / \omega^{2}$ power-law decay. In our calculations we shall assume that $1 / \ell \ll v / D$, meaning that the motion is diffusive also on the spatial scale $\ell$.

For small $k$, meaning $k \leq k_{\perp}$, the transverse term cannot be ignored, and we nolonger can use the approximation (D.3). We can define a time scale $\tau_{\perp}$ that correspond to $k_{\perp}$. It equals $\mathbf{d} / v$ for ballistic motion, $t$ for diffusive motion, and $\tau_{\text {erg }}$ for ergodic motion. For a given small $k$, the function $P(k, \tau)$ is concentrated within $\tau<\tau_{\perp}$, since $P_{\|}$and $P_{\perp}$ are not identical there. Consequently

$$
P(k, \omega) \approx \tau_{\perp} \cdot P(k) \text { for } \omega<1 / \tau_{\perp} \text { in the small } k \text { regime }
$$

The function $P_{\perp}(\mathbf{k})$ is the FT of $P_{\perp}(\mathbf{r})$, and therefore we have for $P(k)=1-P_{\perp}(\mathbf{k})$ the following small $k$ approximation

$$
P(k) \approx \frac{1}{2} r_{\perp}^{2} k^{2}=\left\{\begin{array}{cc}
\frac{1}{2} \mathbf{d}^{2} k^{2} & \text { for ballistic motion } \\
D t \cdot k^{2} & \text { for diffusive motion } \\
\frac{1}{2} L^{2} k^{2} & \text { for ergodic motion }
\end{array}\right.
$$

For large $k$ we have $P(\mathbf{k}) \approx 1$, as implied by (D.3) and the definition (D.2).

\section{Appendix E. Friction Constant For Electrons in Metal}

The effect of electron-electron Coulomb interaction can be analyzed by considering the motion of a single electron under the influence of a fluctuating electrostatic potential $\mathcal{U}(\mathbf{x}, t)$ that is created by the other electrons. Thus the electron experience an interaction with a fluctuating field that is characterized by $\left\langle\mathcal{U}\left(\mathrm{x}^{\prime \prime}, t^{\prime \prime}\right) \mathcal{U}\left(\mathrm{x}^{\prime}, t^{\prime}\right)\right\rangle$. It is well known, using fluctuation-dissipation theorem, that for diffusive electrons the corresponding fluctuation spectrum is

$$
\tilde{w}(\mathbf{k}) \phi(\omega)=\frac{e^{2}}{\sigma_{d}} \cdot \frac{1}{k^{2}} \omega \hbar \operatorname{coth}\left(\frac{\hbar \omega}{2 k_{B} T}\right) \quad \text { for } \omega<\frac{1}{\tau_{c}}, \quad|k|<\frac{1}{\ell}
$$

The ohmic behavior is cut-off by the Drude collision frequency $1 / \tau_{c}$. The elastic mean free path is $\ell=v \tau_{c}$, where $v$ is the Fermi velocity. For $k<1 / \ell$ the power spectrum $P(k, \omega)$ of the diffusive motion is concentrated below the Drude cutoff frequency. 
The ballistic regime $1 / \ell<k$ is of no interest because its contribution is suppressed due to the Drude cutoff. One observes that these fluctuations corresponds to the generalized DLD model (15) with $\sigma=d-2$. Having observed that the mean free path is the physical (effective) cutoff for the spatial fluctuations (this statement is true for $\sigma<4$ ), it follows from the convention $\left|w^{\prime \prime}(0)\right|=1$ that the friction constant (up to dimensionless factor of order unity) is given by the expression

$$
\eta=\frac{e^{2}}{\sigma_{d}}\left(\frac{1}{\ell}\right)^{d}=\frac{1}{D} \Delta_{\ell}
$$

Here $e$ is the charge of an electron, and $\sigma_{d}$ is the conductivity defined for $d=1,2,3$ dimensions. The last equality is obtained by using Einstein relation in order to express $\sigma_{d}$ in terms of $D$. The notation $\Delta_{\ell}$ stand for the mean level spacing within a cube whose volume is $\ell^{d}$.

\section{References}

[1] M. Wilkinson, J. Phys. A: Math. Gen. 21, 4021 (1988). C. Jarzynski, Phys. Rev. Lett. 74, 2937 (1995).

[2] D. Cohen, Phys. Rev. Lett. 78, 2878 (1997); Phys. Rev. E 55, 1422 (1997).

[3] A.O. Caldeira and A.J. Leggett, Physica 121 A, 587 (1983). H. Grabert, P. Schramm and G.L. Ingold, Phys. Rep. 168, 115 (1988).

[4] R.P. Feynman and F.L. Vernon Jr., Ann. Phys. (N.Y.) 24, 118 (1963).

[5] A. Bulgac, G.D. Dang and D. Kusnezov, nucl-th/9711021.

[6] D. Cohen, Phys. Rev. A 44, 2292 (1991).

[7] Y. Imry Introduction to Mesoscopic Physics (Oxford Univ. Press 1997), and references therein.

[8] P. Mohanty, E.M. Jariwala and R. A. Webb. Phvs. Rev. Lett. 77, 3366 (1997). D.S. Golubev and A.D.Zaikin, cond-mat/9710079. cond-mat/9712203.

[9] D. Cohen and Y. Imry, cond-mat/9807038.

[10] D. Cohen and S. Fishman, Phys. Rev. A 39, 6478 (1989).

[11] K. Alder and A. Winther Electromagnetic Excitation, Theory of Coulomb Excitation With Heavy Ions (North Holand 1975).

[12] I thank Uzy Smilansky for pointing out this observation.

[13] F. Oppen and A. Stern, cond-mat/9611079.

[14] S. Chakravarty and A. Schmid, Phys. Rep. 140, 193 (1986). 